\title{
Cirugía de osteo-odonto-queratoprótesis modificada
}

\section{Modified osteo-odonto-keratoprosthesis surgery}

\author{
Christian R. Fau-Fuentes* \\ Fundación Oftalmológica 2020, Centro Adherente Cochrane, Las Condes, Santiago, Chile
}

Paciente varón de 31 años de edad que perdió la visión en un accidente laboral en el que se quemó con el vapor de una gran olla a presión que explotó. Todo su cuerpo resultó quemado, al igual que ambos ojos (Fig. 1), la agudeza visual preoperatoria era solo visualización de proyección de luz.

Este caso corresponde a la primera cirugía realizada en América mediante la técnica osteo-odonto-queratoprótesis modificada (MOOKP) en cooperación con la OOKP Foundation Rome, realizada en Santiago, Chile, en el Hospital Clínico de la Universidad de Chile en el año 2008.

La técnica de osteo-odonto-queratoprótesis (OOKP) fue desarrollada por el Dr. Benedetto Strampelli en 1964, posteriormente, fue perfeccionada mediante modificaciones hechas por el Prof. Dr. GianCarlo Falcinelli. Esta técnica es reconocida y aceptada en el mundo, y se encuentra certificada mediante El protocolo Roma-Viena, publicado en la revista Cornea en el año 2005'.

La MOOKP es un tipo de cirugía oftalmológica que permite recuperar la visión de un ojo ciego cuando los trasplantes de córnea han fallado, o cuando el trasplante de córnea no puede practicarse. La técnica quirúrgica de la MOOKP corresponde a un trasplante autólogo, en el cual se utiliza una lámina osteodental, extraída del maxilar superior, como soporte biológico para un cilindro de polimetilmetacrilato (PMMA), previamente calculado mediante ecografía, por ende específico para cada paciente,

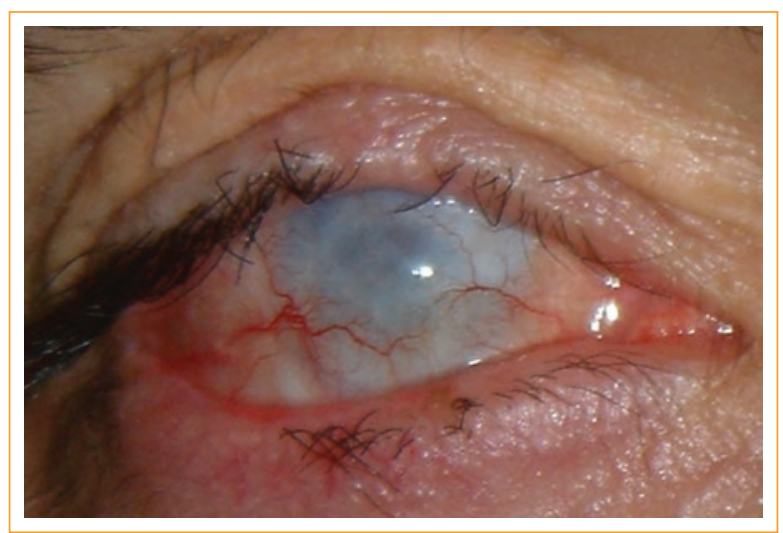

Figura 1. Muestra la condición en la cual quedo el ojo derecho del paciente.

el cual después de un periodo de 2 a 3 meses de integración y vascularización bajo la piel, es colocado sobre la superficie corneal de un globo ocular, previamente preparado para estos fines, lo que permite el libre paso de la luz y, por consiguiente, la recuperación de la visión, con un campo visual de más o menos $40^{\circ}$. Todo ello permite el desarrollo de una vida normal a estos pacientes, y los saca de su condición de ciegos (Fig. 2).

El resultado del paciente a los 3 meses de terminadas todas las etapas de la cirugía fue de 20/30, con corrección de +0.75 esfera de lejos, y Jaeger 7 de cerca, con corrección de +4.00 esfera.
Disponible en internet: 01-07-2019 Rev Mex Oftalmol. 2018;93(4):209-210 www.rmo.com. mx
(n) cencia CC BY-NC-ND
Fecha de recepción: 14-04-2018

Fecha de aceptación: 28-05-2018 DOI: 10.24875/RMO.M18000041 


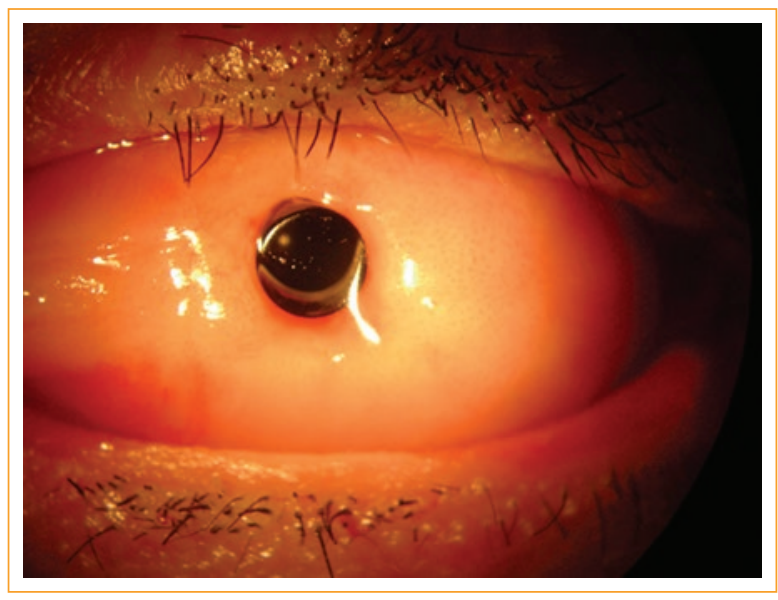

Figura 2. Muestra el resultado quirúrgico de MOOKP en el ojo izquierdo del paciente 3 meses después de terminadas todas las etapas de la cirugía.

\section{Responsabilidades éticas}

Protección de personas y animales. Los autores declaran que los procedimientos seguidos se conformaron a las normas éticas del comité de experimentación humana responsable y de acuerdo con la Asociación Médica Mundial y la Declaración de Helsinki.

Confidencialidad de los datos. Los autores declaran que han seguido los protocolos de su centro de trabajo sobre la publicación de datos de pacientes.

Derecho a la privacidad y consentimiento informado. Los autores han obtenido el consentimiento informado de los pacientes y/o sujetos referidos en el artículo. Este documento obra en poder del autor de correspondencia.

\section{Conflicto de intereses}

Los autores declaran que no existe conflicto de intereses.

\section{Bibliografía}

1. Hille K, Grabner G, Liu C, Colliardo P, Falcinelli G, Taloni M, et al. Standards for Modified Osteoodontokeratoprosthesis (OOKP) Surgery According to Strampelli and Falcinelli: The Rome-Vienna Protocol. Cornea. 2005;24(8):895-908. 